\title{
THE EFFECT OF
}

\section{METHOTREXATE-LOADED BONE CEMENT ON LOCAL DESTRUCTION BY THE VX ${ }_{2}$ TUMOUR}

\author{
H. M. WANG, S. CRANK, G. OLIVER, C. S. B. GALASKO \\ From the University of Manchester, England
}

$\mathbf{P}$

revious studies have shown that the activity of the cytostatic drug methotrexate (MTX) embedded in acrylic cement is not affected by thermal changes in the cement. MTX is slowly released from the cement for several months and remains biologically active throughout this period. Our aim was to determine whether MTX embedded in cement would control the local growth of a tumour.

In 15 rabbits we injected $0.1 \mathrm{ml}$ of $\mathrm{VX}_{2}$ tumour suspension into the proximal tibia. At 3, 5, 7, 10 and 14 days three animals were killed and the tibiae removed and examined histologically. With increasing growth of the $\mathrm{VX}_{2}$ carcinoma there was increased bone destruction and a rise in the numbers of osteoclasts, but after 14 days the numbers of osteoclasts had decreased.

We then injected $\mathrm{VX}_{2}$ into the tibiae of another 45 rabbits. After 5 days most of the tumour was curetted out and the defect filled with cement containing either $0 \mathrm{~g}, 0.1 \mathrm{~g}, 0.5 \mathrm{~g}, 1.0 \mathrm{~g}$ or $2.0 \mathrm{~g}$ MTX/40 g cement. The rabbits were divided into three groups and killed at 3 , 7 or 10 days after implantation of cement. The number of osteoclasts and the amount of bone destruction were measured in each tibia. In all three groups bone destruction and osteoclast proliferation were markedly decreased with higher doses of MTX, but bone destruction was not eliminated.

Our findings show that in the higher doses used, which were not toxic to the animal, MTX-embedded cement may be of value in minimising the amount of tumour-induced osteolysis and may be a useful adjunct in the surgical management of pathological fractures.

J Bone Joint Surg [Br] 1996;78-B:14-17.

Received 28 April 1995; Accepted 21 June 1995

H. M. Wang, MD, PhD, Research Fellow

S. Crank, MSc, Research Biochemist

G. Oliver, Research Assistant

C. S. B. Galasko, MSc, ChM, FRCS, FRCS Ed, Professor of Orthopaedic Surgery

Department of Orthopaedic Surgery, University of Manchester, Clinical Sciences Building, Hope Hospital, Eccles Old Road, Salford, Manchester M6 8HD, UK

Correspondence should be sent to Professor C. S. B. Galasko.

(C)1996 British Editorial Society of Bone and Joint Surgery

0301-620X/96/11107\$2.00
Pathological and impending fractures, secondary to metastatic cancer, are usually treated by internal fixation, often supplemented by methylmethacrylate. In other cases such as pathological fractures of the proximal femur, the lesion is treated by replacement arthroplasty which is cemented in place. Patients are given postoperative radiotherapy and adjuvant endocrine or chemotherapy depending on the primary tumour, in an attempt to keep the tumour under control. Progressive growth of the tumour leads to further osteolysis, loosening and failure of the implant.

Our aim was to determine whether methotrexate (MTX) embedded in the cement would help to control local growth of the tumour. Previous experiments in vitro have shown that cytostatic drugs such as MTX are released from acrylic cement for at least four to six months. The released MTX remains biologically active throughout this period (Hernigou et al 1989; Marshall et al 1990; Wang et al 1995), and the addition of MTX does not appear to alter the mechanical properties of Palacos- $\mathrm{R}$ cement. It has been shown in the $\mathrm{VX}_{2}$ carcinoma rabbit model that the local administration of MTX-loaded cement reduced the number of pulmonary metastases with no detectable toxic effect (Wang et al 1995). Administration of MTX-loaded Palacos-R acrylic cement may reduce the amount of tumour-induced local bone destruction.

Most tumour-induced osteolysis is mediated by osteoclasts (Galasko 1976, 1982; Galasko and Bennett 1976). We therefore chose the number of osteoclasts and the area of bone destruction as criteria to assess the effectiveness of the released MTX.

\section{MATERIALS AND METHODS}

To estimate the optimum period after implantation of the tumour for this investigation, we inoculated 15 rabbits with $0.1 \mathrm{ml}$ of $\mathrm{VX}_{2}$ tumour cell suspension $\left(3 \times 10^{6}\right.$ cells $\left./ \mathrm{ml}\right)$ into the proximal tibia through a drill hole in the cortex. At each of 3, 5, 7, 10 and 14 days after inoculation three rabbits were killed. The tibiae were removed, fixed in $10 \%$ formalin, decalcified in 10\% EDTA and embedded in Paramat wax; $7 \mu \mathrm{m}$ sections were cut and stained with haematoxylin and eosin. The number of osteoclasts for each section were counted in 1000 random sites at a magnification of $\times 1000$ using a cross-hatched eyepiece graticule.

We measured tumour-mediated bone destruction at a magnification of $\times 400$ with the cross-hatched eyepiece 
graticule by determining the area in which tumour replaced cortical bone (excluding soft-tissue replacement or tumour in the medullary cavity). The area of normal bone was measured by the same method. The measurement was carried out within a defined area between a proximal line, taken just below the subchondral proximal tibia, and a distal line, $2.5 \mathrm{~cm}$ below (covering most of the length of the rabbit tibia). The areas of both normal bone and tumourreplaced bone were determined at 100 random sites in the cortical bone. The tumour area was expressed as the percentage of normal bone plus the tumourous area per section.

No areas of bone destruction could be detected in the 3-day specimens, and after 7, 10 and 14 days the amount of osteolysis could not be accurately measured as tumour spread was too extreme. We therefore chose five days after tumour inoculation as the time for assessment of the effect of MTX-loaded bone cement on local tumour-mediated osteolysis.

We then injected 45 mature Half-lop rabbits with $0.1 \mathrm{ml}$ of $\mathrm{VX}_{2}$ tumour cell suspension $\left(3 \times 10^{6}\right.$ cells $\left./ \mathrm{ml}\right)$ in the proximal tibia as described above. At five days each rabbit had Palacos-R bone cement implanted. Under sterile conditions the proximal tibia was exposed through a $2.5 \mathrm{~cm}$ incision and a window, approximately $5 \times 8 \mathrm{~mm}$, was made in the cortex of the tibia above the tumour. Most of the $\mathrm{VX}_{2}$ tumour was curetted out and the defect was filled with cement containing either $0 \mathrm{~g}, 0.1 \mathrm{~g}, 0.5 \mathrm{~g}, 1.0 \mathrm{~g}$ or $2.0 \mathrm{~g}$ MTX/40 g cement. The wound was washed with saline and closed with interrupted 5/0 sutures. Analgesic and antibiotic injections were given postoperatively and sodium bicarbonate $(1 \%)$ was added to the drinking water to prevent renal damage.

The rabbits were studied in three groups of 15; one group was killed at each of 3, 7 and 10 days after implantation of cement. Three rabbits died because of wound infection leaving 42 for final assessment.

At death the tibiae were removed, fixed in $10 \%$ formalin, embedded in methylmethacrylate resin and $7 \mu \mathrm{m}$ sections obtained using a LKB 2260 Macrotome. These were stained with toluidine blue and Von Kossa stain. The number of osteoclasts and the amount of destruction for each section were measured as described above.

\section{RESULTS}

Table I shows the number of osteoclasts and the amount of tumour-induced osteolysis. The mean number of osteoclasts increased from $25 \pm 3$ to $75 \pm 6 / 1000$ random sites between 3 and 7 days after tumour inoculation. With increasing growth of the $\mathrm{VX}_{2}$ carcinoma and increasing bone destruction the number decreased to $45 \pm 5$ at 14 days after $\mathrm{VX}_{2}$ inoculation. The mean area of bone destruction rapidly increased over the 14 days after tumour inoculation.

In most of the 42 processed tibiae a thin layer of necrotic bone was seen at the endosteal surface. Otherwise all other
Table I. The number of osteoclasts (mean \pm SD per 1000 random sites) and area of bone destruction (percentage; mean $\pm \mathrm{SD}$ ) after $\mathrm{VX}_{2}$ inoculation

\begin{tabular}{lll}
\hline $\begin{array}{l}\text { Days after } \mathbf{V X} \mathbf{X}_{\mathbf{2}} \\
\text { inoculation }\end{array}$ & Number of osteoclasts & Area of bone destruction \\
\hline 3 & $25 \pm 3$ & 0 \\
5 & $40 \pm 5$ & $0.24 \pm 0.14$ \\
7 & $75 \pm 6$ & $1.33 \pm 0.64$ \\
10 & $61 \pm 3$ & $4.43 \pm 1.45$ \\
14 & $45 \pm 5$ & $13.65 \pm 1.35$ \\
\hline
\end{tabular}

Table II. The number of osteoclasts (mean \pm SD per 1000 random sites) and area of bone destruction (percentage; mean $\pm \mathrm{SD}$ ) after implantation of MTX-loaded acrylic cement in various dosages

\begin{tabular}{llcc}
\hline $\begin{array}{l}\text { Days after } \\
\text { cement } \\
\text { implantation }\end{array}$ & $\begin{array}{l}\text { Dose of MTX } \\
\text { /40 g cement }\end{array}$ & Number of osteoclasts & $\begin{array}{l}\text { Area of bone } \\
\text { destruction }\end{array}$ \\
\hline 3 & 0 & $3 \pm 1$ & $0.73 \pm 0.13$ \\
& 0.1 & $3 \pm 1$ & $0.57 \pm 0.13$ \\
& 0.5 & $3 \pm 1$ & $0.63 \pm 0.04$ \\
& 1.0 & $1.67 \pm 1.15$ & $0.49 \pm 0.05$ \\
& 2.0 & $3.33 \pm 1.16$ & $0.36 \pm 0.06$ \\
7 & 0 & $5.5 \pm 0.71$ & $5.62 \pm 1.10$ \\
& 0.1 & $5.0 \pm 1.41$ & $5.54 \pm 1.11$ \\
& 0.5 & $4.33 \pm 0.58$ & $3.60 \pm 0.64$ \\
& 1.0 & $2.33 \pm 0.58$ & $0.86 \pm 0.26$ \\
10 & 2.0 & $2.67 \pm 0.58$ & $0.53 \pm 0.10$ \\
& 0 & $11.33 \pm 2.52$ & $12.88 \pm 1.41$ \\
& 0.1 & $8.0 \pm 1.0$ & $11.07 \pm 1.75$ \\
& 0.5 & $4.33 \pm 0.58$ & $8.88 \pm 0.32$ \\
& 1.0 & $3.5 \pm 0.71$ & $6.83 \pm 0.88$ \\
& 2.0 & $3.33 \pm 0.58$ & $1.98 \pm 0.42$ \\
\hline
\end{tabular}

osteocytes were normal and viable, even those in contact with cement containing high doses of MTX. The radiopaque bone cement filled most of the medullary cavity. The histological appearance of the osteoclasts was normal and they were usually located very close to the bone and away from the margins of the tumour.

The number of osteoclasts and the percentage area of bone destruction in the groups with implanted cement at 3, 7 and 10 days are shown in Table II. There was no difference in the number of osteoclasts in the control (without MTX) and the $0.1 \mathrm{~g}$ and $0.5 \mathrm{~g}$ MTX/40 $\mathrm{g}$ experimental groups at 3 and 7 days, but there were fewer osteoclasts in the $1 \mathrm{~g}$ MTX/40 $\mathrm{g}$ cement group. After 10 days there was a reduction in the number of osteoclasts in all the MTX-loaded cement sections when compared with the control group. In the latter, however, the number of osteoclasts was $3 \pm 1,5.5 \pm 0.71$ and $11.33 \pm 2.52 / 1000$ random sites at 3,7 and 10 days respectively, showing that osteoclast-mediated osteolysis was continuing.

The area of cortical bone destruction was reduced in all the MTX experimental groups when compared with the control groups. The most marked decrease was in the $2.0 \mathrm{~g}$ MTX/40 g cement group at 7 and 10 days.

Histological examination of the sections also showed that in the control groups most of the cancellous bone had 
been replaced by tumour, but in the $2.0 \mathrm{~g} \mathrm{MTX} / 40 \mathrm{~g}$ cement group considerable cancellous bone could still be seen. It was difficult to assess the area of cancellous bone destruction by this method of microscopic analysis.

\section{DISCUSSION}

The amount and rate of bone resorption depend on the malignancy and activity of the tumour cells, the number of tumour cells inoculated and the presence of humoral factors. The basic characteristics of the $\mathrm{VX}_{2}$ carcinoma used in our study are similar to those observed by Enneking and Flynn (1968) and Galasko (1975) who showed that cortical bone destruction could not be detected histologically until 7 days after tumour implantation. Our control study showed that cortical bone destruction began on day 5 and that $1.33 \%$ of the cortical bone had been destroyed by day 7 . Cerino, King and Janes (1963) suggested that the rate of bone destruction was probably related to the concentration of inoculated tumour cells, but this was not quantified.

The number of osteoclasts in bone tissue may indirectly reflect the growth and activity of the $\mathrm{VX}_{2}$ tumour and hence the degree of tumour-induced osteolysis. In our study, cortical bone destruction was first seen at day 5. It then increased as the number of osteoclasts increased until day 7. When the tumour reached the endosteal surface of the cortex (days 7 to 10) the osteoclasts were seen in large lacunae within the cortical bone. By days 10 to 14 there was extensive bone destruction and the tumour had enveloped residual trabeculae of bone. Although the number of osteoclasts had considerably decreased by day 14 , cortical bone destruction continued to increase, confirming that osteolysis was continuing. These observations confirm the findings of Galasko (1976).

The number of osteoclasts in the $0 \mathrm{~g}$ MTX/40 $\mathrm{g}$ cement group was less than in the control group $\left(\mathrm{VX}_{2}\right.$ tumour alone), at a similar time interval, probably because of the removal of the tumour by curettage.

In animals killed at 10 days after implantation of cement the number of osteoclasts in the $0 \mathrm{~g}$ MTX/40 $\mathrm{g}$ cement group was greater than in the other control groups indicating that osteoclast recruitment and osteolysis were proceeding unchecked.

The progressive bone resorption observed may be related to the amount of osteoclast-stimulating humoral factors secreted by the $\mathrm{VX}_{2}$ tumour cells. Osteoclasts may also have released growth factors embedded in the bone matrix as the bone resorption progressed. These may have stimulated the tumour which in turn stimulated further osteoclast recruitment and further bone destruction. Because of the release of these growth factors the tumour may grow more rapidly in bone, once it is established, than in other sites.

There have been many studies on the reaction of bone to acrylic cement. In several studies, a thin layer of necrotic bone was found at the cement-bone interface which was thought to be due either to the heat produced by the curing cement (Andersson, Freeman and Swanson 1972; Miller 1972; Hadjari et al 1990) or to the toxic effect of the cement monomer (Jefferiss, Lee and Ling 1975; Linder 1977; Reckling and Dillon 1977).

In our study the formation of a thin layer of necrotic bone at the tibial endosteal surface was not related to the dose of MTX incorporated in the bone cement. Histological examination also showed that the local osteocytes were unaffected. It is possible that the amount of released MTX was not at a toxic level. The long-term application of local chemotherapy did not cause wound-healing problems but skin necrosis and bitten wounds (perhaps from irritation) were seen in three rabbits and led to their premature death. We were unable to determine whether these injuries were a response to a local reaction to MTX.

Most investigators have acknowledged the presence of a fibrous membrane at the cement-bone interface. Goldring et al (1983) found that this membrane could produce large amounts of prostaglandin $\mathrm{E}_{2}$ and collagenase, both known to be associated with osteolysis. Goodman and Chin (1990) suggested that acrylic cement particles, phagocytosed by foreign-body giant cells and then extruded into the extracellular compartment, may be implicated in the formation of the membrane and bone lysis. The longest observation period in our study was 10 days and this was probably too short for the formation of a fibrous membrane. Charnley (1970) noted that a bone specimen, obtained 17 days after cement implantation, showed no fibrous membrane at the cement-bone interface.

Our findings show that at all the implantation times cortical bone destruction was markedly decreased by the higher doses of MTX. Microscopic analysis also indicated that MTX minimised the area of cancellous bone destruction in the high-dose MTX groups. In the control groups most cancellous bone had been replaced by tumour.

Although the local administration of MTX-loaded acrylic cement was effective in reducing the amount of local bone destruction, it did not stop progressive bone lysis, even in the highest-dose MTX group. Histological examination also showed that the number of $\mathrm{VX}_{2}$ cells increased with time. In a rat sarcoma model, Wu et al (1990) showed a tumour suppression rate of 54\% using adriamycin-impregnated bone cement and ceramic implants to fill the bony defects. The implant gave a high concentration of drug locally, but a low systemic concentration.

In the $\mathrm{VX}_{2}$ tumour rabbit model, the local administration of MTX at the highest concentration used in our experiment (2.0 g MTX/40 g cement) markedly reduced the amount of local bone destruction, but did not totally destroy the tumour cells. It is likely therefore that factors which recruited and stimulated osteoclast proliferation may still have been released by the $\mathrm{VX}_{2}$ cells and the use of MTXloaded cement would not completely stop the tumourinduced osteolysis. It would reduce, however, the amount of continuing osteolysis. Our results suggest that, in the clinical situation, the addition of MTX to cement in a 
concentration of $2.0 \mathrm{~g}$ MTX/40 g cement may minimise the amount of continuing tumour-induced osteolysis and reduce the risk of loosening and implant failure. The addition of MTX to cement may therefore be a useful adjunctive treatment in association with postoperative radiotherapy or chemotherapy.

No benefits in any form have been received or will be received from a commercial party related directly or indirectly to the subject of this article.

\section{REFERENCES}

Andersson GBJ, Freeman MAR, Swanson SAV. Loosening of the cemented acetabular cup in total hip replacement. J Bone Joint Surg [Br] 1972;54-B:590-9.

Cerino LE, King BJ, Janes JM. Growth of VX-2 carcinoma in bone. Proc Mayo Clin 1963;38:484-9.

Charnley J. The reaction of bone to self-curing acrylic cement: a long-term histological study in man. J Bone Joint Surg [Br] 1970;52-B:340-53.

Enneking WF, Flynn L. Effects of VX-2 carcinoma implanted in bone in rabbits. Cancer Res 1968;28:1007-13.

Galasko CSB. The pathological basis for skeletal scintigraphy. $J$ Bone Joint Surg [Br] 1975;57-B:353-9.

Galasko CSB. Mechanisms of bone destruction in the development of skeletal metastases. Nature 1976;263:507-8.

Galasko CSB. Mechanisms of lytic and blastic metastatic disease of bone. Clin Orthop 1982;169:20-7.

Galasko CSB, Bennett A. Relationship of bone destruction in skeletal metastases to osteoclast activation and prostaglandins. Nature 1976;263:508-10.
Goldring SR, Schiller AL, Roelke M, et al. The synovial-like membrane at the bone-cement interface in loose total hip replacements and its proposed role in bone lysis. J Bone Joint Surg [Am] 1983;65-A:575-84.

Goodman SB, Chin RC. Prostaglandin $\mathrm{E}_{2}$ levels in the membrane surrounding bulk and particulate polymethylmethacrylate in the rabbit tibia: a preliminary study. Clin Orthop 1990;257:305-9.

Hadjari M, Reindel ES, Kitabayashi L, Convery FR. The effects of cement penetration on the bone-cement interface membrane. In: Trans 36th Annual Meeting, Orthopaedic Research Society, New Orleans, 1990:439.

Hernigou P, Thiéry JP, Benoit J, et al. Methotrexate diffusion from acrylic cement: local chemotherapy for bone tumours. J Bone Joint Surg [Br] 1989;71-B:804-11.

Jefferiss CD, Lee AJC, Ling RMS. Thermal aspects of self-curing polymethylmethacrylate. J Bone Joint Surg [Br] 1975;57-B:511-8.

Linder L. Reaction of bone to the acute chemical trauma of bone cement. J Bone Joint Surg [Am] 1977;59-A:82-7.

Marshall GJ, Kirchen ME, Lee JH, Menendez LA. The effect of methotrexate eluted from bone cement on giant cell tumour lines in vitro. In: Trans 36th Annual Meeting, Orthopaedic Research Society, New Orleans, 1990:573.

Miller AJ. Late fracture of the acetabulum after total hip replacement. J Bone Joint Surg [Br] 1972;54-B:600-6.

Reckling FW, Dillon WL. The bone-cement interface temperature during total joint replacement. J Bone Joint Surg [Am] 1977;59-A:80-2.

Wang HM, Galasko CSB, Crank S, Oliver G, Ward CA. Methotrexate loaded acrylic cement in the management of skeletal metastases: biomechanical, biological and systemic effect. Clin Orthop 1995;312:173-86.

Wu YG, Wang TY, Ma YZ, Sun SZ. Experimental research on the use of antineoplastic drug with a bone implant. Int Orthop 1990;14:387-91. 